\title{
LINEAR CONTROL SYSTEMS ON UNBOUNDED TIME INTERVALS AND INVARIANT MEASURES OF ORNSTEIN-UHLENBECK PROCESSES IN HILBERT SPACES*
}

\author{
MARCO FUHRMAN ${ }^{\dagger}$ AND ANNA MARIA PAGANONI ${ }^{\dagger}$
}

Abstract. We consider linear control systems in a Hilbert space over an unbounded time interval of the form

$$
y_{\alpha}^{\prime}(t)=(A-\alpha I) y_{\alpha}(t)+B u(t), \quad t \in(-\infty, T],
$$

with bounded control operator $B$, under appropriate stability assumptions on the operator $A$. We study how the space of states reachable at time $T$ depends on the parameter $\alpha \geq 0$. We apply the results to study the dependence on $\alpha$ of the Cameron-Martin spaces of the invariant measures of the Ornstein-Uhlenbeck processes $X_{\alpha}$ defined by the equation driven by the Wiener process $W$ :

$$
d X_{\alpha}(t)=(A-\alpha I) X_{\alpha}(t) d t+B d W(t), \quad t \geq 0 .
$$

Key words. infinite dimensional control systems, reachability, Ornstein-Uhlenbeck process

AMS subject classifications. Primary, 93C25, 37L55; Secondary, 93B03, 60H99

DOI. $10.1137 / \mathrm{S} 0363012902414652$

1. Introduction. The aim of this paper is to study some controllability properties of a linear control system in a Hilbert space $H$ over an unbounded time interval and to apply these results to study the behavior of the Cameron-Martin space of the invariant measure for a class of Ornstein-Uhlenbeck stochastic processes in $H$ under perturbation.

Let us consider a linear control system in $H$ of the form

$$
y^{\prime}(t)=A y(t)+B u(t), \quad t \in(-\infty, T],
$$

for $t$ varying in a fixed unbounded time interval $(-\infty, T]$, where $A$ is the infinitesimal generator of a strongly continuous semigroup of operators, $B$ is a bounded operator from another Hilbert space $U$ to $H$, and $u$ is a control, which will always be assumed to belong to the space $L^{2}(-\infty, T ; U)$ of square summable functions from $(-\infty, T]$ to $U$. The value of $T$ is irrelevant in most of what follows, and we could even replace $T$ by zero; nevertheless, we will keep the present, slightly more general notation. For the moment we assume for simplicity that $A$ is exponentially stable, although this assumption will be relaxed in the following sections. Then the solution of (1.1) can be defined in a standard way for every control $u$. The initial condition at $-\infty$ is assumed to be zero. One defines in an obvious way the space of states reachable at time $T$ over the interval $(-\infty, T]$, which is denoted by $\mathcal{K}_{\infty}$. By straightforward extensions of the results on a finite time interval, this space can be characterized as the image of the square root of the operator $Q_{\infty}$ defined by

$$
Q_{\infty} h=\int_{0}^{\infty} e^{t A} B B^{*} e^{t A^{*}} h d t, \quad h \in H
$$

Thus $\mathcal{K}_{\infty}=i m Q_{\infty}^{1 / 2}$. Clearly, this space does not depend on $T$.

\footnotetext{
${ }^{*}$ Received by the editors September 18, 2002; accepted for publication (in revised form) May 6, 2003; published electronically November 14, 2003.

http://www.siam.org/journals/sicon/42-5/41465.html

${ }^{\dagger}$ Dipartimento di Matematica, Politecnico di Milano, Piazza Leonardo da Vinci 32, 20133 Milano, Italy (marco.fuhrman@polimi.it, annamaria.paganoni@mate.polimi.it).
} 
One of the main results of this paper is a precise description of the behavior of the space of reachable states for the class of perturbed control systems

$$
y_{\alpha}^{\prime}(t)=(A-\alpha I) y_{\alpha}(t)+B u(t), \quad t \in(-\infty, T],
$$

when the parameter $\alpha$ ranges over $[0, \infty)$.

We note that the system (1.2) can be reduced to system (1.1) by the transformation $y(t)=e^{\alpha t} y_{\alpha}(t)$. This transformation is sometimes called the "exponential shift." For control systems on a finite time interval this argument shows that the space of reachable states does not depend on $\alpha$. We note, however, that the exponential shift maps the control $u$ to the function $t \mapsto e^{\alpha t} u(t)$, which does not necessarily belong to the space $L^{2}(-\infty, T ; U)$ of square summable controls, due to the fact that the time interval $(-\infty, T]$ is unbounded. Keeping the space of controls fixed, the problem arises whether the space of reachable sets may change with $\alpha$. One can also study a related problem, namely, whether a state which is reachable on $(-\infty, T]$ can also be reached over a finite time interval $[T-r, T]$ for some $r>0$.

In Theorem 4.1 we give an answer to both questions. Somewhat imprecisely, the situation is as follows: denoting by $\mathcal{K}_{\infty}^{\alpha}$ the space of reachable states for the perturbed systems (1.2), it is clear that

$$
0 \leq \alpha \leq \beta \quad \text { implies } \quad \mathcal{K}_{\infty}^{\alpha} \supset \mathcal{K}_{\infty}^{\beta},
$$

possibly with equality. It can happen that $\mathcal{K}_{\infty}^{\alpha}$ are strictly decreasing for all $\alpha \geq 0$. Or it can happen that $\mathcal{K}_{\infty}^{\alpha}$ are strictly decreasing for $\alpha$ less or equal to some critical value $\alpha_{0}$, and then they remain unchanged for $\alpha>\alpha_{0}$; the case $\alpha_{0}=0$ may occur. Another case can also happen, namely, that $\mathcal{K}_{\infty}^{\alpha}$ do not depend on $\alpha$. Finally, the states in $\mathcal{K}_{\infty}^{\alpha}$ can be reached in a finite time interval precisely when a small change of $\alpha$ does not change the reachable set. See Theorem 4.1 for precise statements. We also relate the critical value $\alpha_{0}$ to the norms of some appropriately defined operators; see Corollary 4.7.

One may ask whether more general perturbations of the original system (1.1) could affect the space $\mathcal{K}_{\infty}$ of reachable states over $(-\infty, T]$. The results described above show the (perhaps surprising) fact that even the spaces $\mathcal{K}_{\infty}^{\alpha}$ are very "sensitive" to perturbations; an arbitrarily small change in the value of $\alpha$ may suffice to change the space $\mathcal{K}_{\infty}^{\alpha}$. Therefore, one expects that more general perturbations of the original system (1.1) will have the same effect, unless very stringent conditions are imposed. That is why we do not address this problem here but rather postpone it to future study.

We devote section 5 to giving examples where the various possibilities described above occur and also to studying some important classes of control systems. For example, we show that the spaces $\mathcal{K}_{\infty}^{\alpha}$ do not depend on $\alpha$ if the system is finitedimensional or exactly null controllable.

In connection with all the properties and problems discussed so far, instead of the set of reachable states one may consider the set of approximately reachable states. In strong contrast with the previous results, the space of approximately reachable states for system (1.2) turns out to be independent of $\alpha$; see section 4.3.

We believe that the previous results have an intrinsic interest, since they address basic structural properties of linear control systems, but our interest in this question arose from some probabilistic motivation, which we now shortly describe. 
Let us consider the following stochastic evolution equation of Itô type:

$$
\left\{\begin{array}{l}
d X(t)=A X(t) d t+B d W(t), \quad t \geq 0 \\
X(0)=X_{0}
\end{array}\right.
$$

where $W$ is a (cylindrical) Wiener process in a filtered probability space $\left(\Omega, \mathcal{F}, \mathbb{P},\left(\mathcal{F}_{t}\right)\right)$ and $X_{0}$ is a gaussian $\mathcal{F}_{0}$-measurable random variable with values in $H$. Assuming the finite trace condition

$$
\text { Trace } Q_{\infty}<\infty
$$

one can show that the equation uniquely defines a gaussian process $X$ and that the centered gaussian measure $\mu$ with covariance operator $Q_{\infty}$ is invariant for the process $X$. For these facts we refer to [8] and to the discussion in section 6 . The process $X$ is called the (nonsymmetric) Ornstein-Uhlenbeck process and has been intensively studied in recent years; see $[3,4,5,6,13,14]$.

Associated to the centered gaussian measure $\mu$ is the so-called Cameron-Martin space; see, e.g., [1]. It is the subspace of $H$ consisting of all vectors $h$ such that the image measure of $\mu$ under the mapping $x \rightarrow x+h, x \in H$, is absolutely continuous with respect to $\mu$. The corresponding Radon-Nikodym density is then called the logarithmic derivative of $\mu$ along $h$. The Cameron-Martin space plays a basic role in the construction of the Sobolev classes of functions over the measure space $(H, \mu)$ (see [1]), in the definition and properties of the associated Dirichlet forms (see [2], $[17]$ ), and in the subsequent constructions needed for the analysis of the stochastic process $X$ (see the references on the Ornstein-Uhlenbeck process cited above). It turns out that the Cameron-Martin space of $\mu$ coincides with $i m Q_{\infty}^{1 / 2}$ and hence with the space of reachable states $\mathcal{K}_{\infty}$ for the control system (1.1). So our results can be applied to study how the Cameron-Martin space behaves under perturbation of the Ornstein-Uhlenbeck process. Thus, for $\alpha \geq 0$, we consider the perturbed processes $X_{\alpha}$ solution of

$$
\left\{\begin{array}{l}
d X_{\alpha}(t)=(A-\alpha I) X_{\alpha}(t) d t+B d W(t), \quad t \geq 0 \\
X_{\alpha}(0)=X_{0}
\end{array}\right.
$$

and we consider the corresponding gaussian invariant measures $\mu_{\alpha}$. Since the CameronMartin space of $\mu_{\alpha}$ coincides with $\mathcal{K}_{\infty}^{\alpha}$, our results give information on the dependence of the Cameron-Martin space of $\mu_{\alpha}$ on $\alpha$.

The plan of the paper is as follows: Section 2 is devoted to some preliminary technical facts. Section 3 contains the standing assumptions and some preliminary results on control systems on unbounded time intervals. Section 4 contains the main results, Theorem 4.1 and Corollary 4.7, while section 5 is devoted to examples. Finally, in section 6 it is shown how to apply results to the Ornstein-Uhlenbeck process.

2. Some notation and technical tools. Let $H$ and $U$ be separable Hilbert spaces, over the real or complex field, with norm and scalar product denoted by $|\cdot|$, $\langle\cdot\rangle$. We use $\|\cdot\|$ to denote the operator norm. Later we will consider $H$ as the state space of a control system and $U$ as the space of control parameters.

We start by recalling a few facts on Hilbert space valued integrals.

Let $I$ be an interval in the real line and $f: I \rightarrow H$ a Borel measurable function. It is well known that $f$ is integrable (in the sense of Bochner) if and only if $\int_{I}|f(t)| d t<$ $\infty$. To allow more generality, in the following we will use the concept of weak integrals, 
and so we are going to recall the definition and some of its elementary properties; for more on this subject the reader may consult, e.g., [9]. We say that $f$ is weakly integrable if $\int_{I}|\langle f(t), h\rangle| d t<\infty$ for every $h \in H$ and there exists a (necessarily unique) element of $H$, denoted $\int_{I} f(t) d t$, such that

$$
\left\langle\int_{I} f(t) d t, h\right\rangle=\int_{I}\langle f(t), h\rangle d t, \quad h \in H .
$$

If $f$ is Bochner integrable, then $\int_{I} f(t) d t$ coincides with the Bochner integral. The definition of weak integrability, as stated above, is slightly redundant, as shown by the following well-known lemma that will be useful in what follows; see, for example, $[10,9]$.

Lemma 2.1. If $f: I \rightarrow H$ is Borel measurable and $\int_{I}|\langle f(t), h\rangle| d t<\infty$ for every $h \in H$, then $f$ is weakly integrable.

In the following sections we will systematically use the following lemma, whose proof can be found in [7] or [21].

Lemma 2.2. Let $H, U, Z$ be Hilbert spaces, and let $A: U \rightarrow H$ and $B: Z \rightarrow H$ be bounded linear operators. Then the inclusion between the images

$$
\text { im } A \subset \operatorname{im} B
$$

holds if and only if there exists $c>0$ such that

$$
\left|A^{*} h\right| \leq c\left|B^{*} h\right|, \quad h \in H .
$$

In this case, denoting by $B^{-1}$ the pseudoinverse of $B$, we have $\left\|B^{-1} A\right\| \leq c$.

In particular, if we define $Q=B B^{*}$, then im $B=i m Q^{1 / 2}$ and, denoting $Q^{-1 / 2}$ the pseudoinverse of $Q^{1 / 2}$, we have $\left\|B^{-1} Q^{1 / 2}\right\|=\left\|Q^{-1 / 2} B\right\|=1$.

3. Assumptions and preliminaries. Throughout the paper the following assumptions are assumed to hold.

HYPOTHESIS 3.1.

(i) $H$ and $U$ are separable Hilbert spaces.

(ii) The operator $A$ is the generator of a strongly continuous semigroup $\left\{e^{t A}, t \geq\right.$ $0\}$ of bounded linear operators in $H . B$ is a bounded linear operator from $U$ to $H$.

(iii) We have

$$
\int_{0}^{\infty}\left|B^{*} e^{t A^{*}} x\right|^{2} d t<\infty, \quad x \in H
$$

We remark that the validity of (3.1) does not imply that the operator $A$ is exponentially stable. Condition (3.1) has received a fair amount of attention in some of the recent literature. At least in the case when $U=\mathbb{R}$ (and therefore $B^{*}$ maps $H$ to $\mathbb{R}$ ), in several cases condition (3.1) is equivalent to a bound of the form $\left|B^{*}\left(s I-A^{*}\right)^{-1}\right|_{H^{*}} \leq c / \sqrt{\operatorname{Re} s}$ for complex $s$ with Re $s>0$; see, for instance, $[15,20,16]$.

Let us consider a control system in $H$ on a finite time interval. We shortly recall some usual definitions and properties, mainly to fix notation. For further details we refer the reader to any treatise on infinite-dimensional control theory, for instance, [7] or $[21]$. 
We will consider a control system on a time interval of length $r>0$, with initial condition $h \in H$, formally:

$$
y^{\prime}(t)=A y(t)+B u(t), \quad t \in[T-r, T], \quad y(T-r)=h .
$$

Here $T$ is an arbitrary fixed real number; the choice of the time interval $[T-r, T]$ allows us to conform with some notation that will be introduced later, when dealing with the unbounded time interval $(-\infty, T]$.

When referring to (3.2), we take as the space of controls the space $L^{2}(T-r, T ; U)$ of all Borel measurable functions $u:[T-r, T] \rightarrow U$ satisfying $\int_{T-r}^{T}|u(t)|^{2} d t<\infty$. $L^{2}(T-r, T ; U)$ will be endowed with its usual Hilbert norm. The solution $y:[T-$ $r, T] \rightarrow H$ of (3.2), or the trajectory corresponding to a control $u$, is defined as

$$
y(t)=e^{(t-T+r) A} h+\int_{T-r}^{t} e^{(t-s) A} B u(s) d s, \quad t \in[T-r, T],
$$

and the space $\mathcal{K}_{r}$ of states reachable from zero in time $r$ (in short, the space of reachable states) is defined as the set of all elements $y(T)$, as $u$ spans the space of controls and $h=0$. The space $\mathcal{K}_{r}$ therefore coincides with the image of the so-called controllability operator $\mathcal{L}_{r}: L^{2}(T-r, T ; U) \rightarrow H$ defined by

$$
\mathcal{L}_{r} u=\int_{T-r}^{T} e^{(T-s) A} B u(s) d s, \quad u \in L^{2}(-\infty, T ; U) .
$$

The gramian operator is defined by

$$
Q_{r} h=\int_{0}^{r} e^{t A} B B^{*} e^{t A^{*}} h d t, \quad h \in H,
$$

and we have $\mathcal{L}_{r} \mathcal{L}_{r}^{*}=Q_{r}$ and $\mathcal{K}_{r}=i m \mathcal{L}_{r}=i m Q_{r}^{1 / 2}$. Clearly, $\mathcal{K}_{s} \subset \mathcal{K}_{r}$ for $0<s<r$.

In the case of a finite time interval considered so far, the assumption (3.1) is not needed, and all the indicated integrals are Bochner integrals.

Now, for fixed $T \in \mathbb{R}$, we consider the following control system in $H$ on the unbounded time interval $(-\infty, T]$ :

$$
y^{\prime}(t)=A y(t)+B u(t), \quad t \in(-\infty, T], \quad y(-\infty)=0 .
$$

This expression is only formal, but now we proceed to giving rigorous definitions. We define a control as an element of $L^{2}(-\infty, T ; U)$, i.e., a Borel measurable function $u:(-\infty, T] \rightarrow U$ satisfying $\int_{-\infty}^{T}|u(t)|^{2} d t<\infty . L^{2}(-\infty, T ; U)$ will be endowed with its usual Hilbert norm. A trajectory of the control system (3.3), corresponding to the control $u$, is by definition the function

$$
y(t)=\int_{-\infty}^{t} e^{(t-s) A} B u(s) d s, \quad t \in(-\infty, T] .
$$

We note that, for every $h \in H$,

$$
\begin{aligned}
\int_{-\infty}^{t}\left|\left\langle e^{(t-s) A} B u(s), h\right\rangle\right| d s & \leq \int_{-\infty}^{t}|u(s)|\left|B^{*} e^{(t-s) A^{*}} h\right| d s \\
& \leq\left(\int_{-\infty}^{t}|u(s)|^{2} d s\right)^{1 / 2}\left(\int_{-\infty}^{t}\left|B^{*} e^{(t-s) A^{*}} h\right|^{2} d s\right)^{1 / 2} \\
& =\left(\int_{-\infty}^{t}|u(s)|^{2} d s\right)^{1 / 2}\left(\int_{0}^{\infty}\left|B^{*} e^{s A^{*}} h\right|^{2} d s\right)^{1 / 2}
\end{aligned}
$$


and the right-hand side is finite by the assumption (3.1). It follows that $y(t)$ is well defined as a weak integral by Lemma 2.1 .

We define the space of reachable states $\mathcal{K}_{\infty}$ as the set of all elements $y(T)$, as $u$ spans the space of controls. The space $\mathcal{K}_{\infty}$ therefore coincides with the image of the operator $\mathcal{L}_{\infty}: L^{2}(-\infty, T ; U) \rightarrow H$ defined as

$$
\mathcal{L}_{\infty} u=\int_{-\infty}^{T} e^{(T-s) A} B u(s) d s, \quad u \in L^{2}(-\infty, T ; U) .
$$

We may call $\mathcal{L}_{\infty}$ the extended controllability operator. The adjoint operator $\mathcal{L}_{\infty}^{*}$ : $H \rightarrow L^{2}(-\infty, T ; U)$ is easily computed: for $h \in H, \mathcal{L}_{\infty}^{*} h$ is the function

$$
\mathcal{L}_{\infty}^{*} h(s)=B^{*} e^{(T-s) A^{*}} h, \quad s \in(-\infty, T] .
$$

In particular, we find

$$
\mathcal{L}_{\infty} \mathcal{L}_{\infty}^{*} h=\int_{-\infty}^{T} e^{(T-s) A} B B^{*} e^{(T-s) A^{*}} h d s=\int_{0}^{\infty} e^{t A} B B^{*} e^{t A^{*}} h d s,
$$

which is a well-defined weak integral. So we can define the extended gramian operator

$$
Q_{\infty} h=\int_{0}^{\infty} e^{t A} B B^{*} e^{t A^{*}} h d t, \quad h \in H
$$

and conclude that

$$
\mathcal{L}_{\infty} \mathcal{L}_{\infty}^{*}=Q_{\infty}
$$

which implies that $Q_{\infty}$ is a bounded nonnegative self-adjoint operator in $H$ and, by Lemma 2.2, that the space $\mathcal{K}_{\infty}$ coincides with the image of $Q_{\infty}^{1 / 2}$.

The reader may note that these are immediate extensions of the corresponding notions for systems on a finite time interval.

4. Perturbed systems: Main result. In this section Hypothesis 3.1 is still in force. Our main concern will be to investigate the behavior of the space of reachable states when the system (3.3) is perturbed by replacing the operator $A$ with $A-\alpha I$, where $\alpha \geq 0$. Note that (3.1) holds with $A$ replaced by $A-\alpha I$ since $\alpha \geq 0$. We still keep $L^{2}(-\infty, T ; U)$ as the space of controls.

Thus we are considering the family of control systems written formally

$$
y_{\alpha}^{\prime}(t)=(A-\alpha I) y_{\alpha}(t)+B u(t), \quad t \in(-\infty, T], \quad y_{\alpha}(-\infty)=0 .
$$

We note that $A-\alpha I$ is the generator of the semigroup $\left\{e^{-\alpha t} e^{t A}, t \geq 0\right\}$. According to the previous definitions, for the perturbed control system (4.1) the space of reachable states $\mathcal{K}_{\infty}^{\alpha}$ is defined as the image of the operator $\mathcal{L}_{\infty}^{(\alpha)}: L^{2}(-\infty, T ; U) \rightarrow H$ given by

$$
\mathcal{L}_{\infty}^{(\alpha)} u=\int_{-\infty}^{T} e^{-\alpha(T-s)} e^{(T-s) A} B u(s) d s, \quad u \in L^{2}(-\infty, T ; U) .
$$

The extended gramian operator for (4.1) is

$$
Q_{\infty}^{(\alpha)} h=\int_{0}^{\infty} e^{-2 \alpha t} e^{t A} B B^{*} e^{t A^{*}} h d t, \quad h \in H
$$


and we have $\mathcal{L}_{\infty}^{(\alpha)}\left(\mathcal{L}_{\infty}^{(\alpha)}\right)^{*}=Q_{\infty}^{(\alpha)}$ and $\mathcal{K}_{\infty}^{\alpha}=i m \mathcal{L}_{\infty}^{(\alpha)}=i m\left(Q_{\infty}^{(\alpha)}\right)^{1 / 2}$ as before. Note that $\mathcal{K}_{\infty}^{0}=\mathcal{K}_{\infty}, \mathcal{L}_{\infty}^{(0)}=\mathcal{L}_{\infty}, Q_{\infty}^{(0)}=Q_{\infty}$. We also note that for $0 \leq \alpha<$ $\beta$ the inequality $Q_{\infty}^{(\beta)} \leq Q_{\infty}^{(\alpha)}$ is obvious, and by Lemma 2.2 this implies $\mathcal{K}_{\infty}^{\beta}=$ $\operatorname{im}\left(Q_{\infty}^{(\beta)}\right)^{1 / 2} \subset i m\left(Q_{\infty}^{(\alpha)}\right)^{1 / 2}=\mathcal{K}_{\infty}^{\alpha}$.

We may also consider the perturbed system on a bounded time interval; formally, for $r>0$,

$$
y_{\alpha}^{\prime}(t)=(A-\alpha I) y_{\alpha}(t)+B u(t), \quad t \in[T-r, T], \quad y_{\alpha}(T-r)=0 .
$$

However, the space of reachable states for this system clearly does not depend on $\alpha$ and is equal to the image of the operators $\mathcal{L}_{r}$ introduced above, since the exponential shift, described in the introduction, leaves the space $L^{2}(T-r, T ; U)$ unchanged.

So far the following inclusions have been (trivially) verified: for $0 \leq \alpha<\beta$ and $0<s<r$ we have

$$
\mathcal{K}_{\infty}^{\alpha} \supset \mathcal{K}_{\infty}^{\beta} \supset \mathcal{K}_{r} \supset \mathcal{K}_{s}
$$

Our main result is the following.

THEOREM 4.1. Assume Hypothesis 3.1. Then one and only one of the following situations occurs.

(i) We have

$$
\mathcal{K}_{\infty}^{\alpha}=\mathcal{K}_{\infty}^{\beta} \quad \text { whenever } \quad 0 \leq \alpha<\beta
$$

Moreover, there exists $r>0$ such that $\mathcal{K}_{r}=\mathcal{K}_{\infty}^{\alpha}$ for every $\alpha \geq 0$.

(ii) There exists $\alpha_{0} \in[0, \infty]$ such that

$$
\mathcal{K}_{\infty}^{\gamma_{1}} \supsetneq \mathcal{K}_{\infty}^{\gamma_{2}} \supsetneq \mathcal{K}_{\infty}^{\alpha_{0}} \supsetneq \mathcal{K}_{\infty}^{\beta_{1}}=\mathcal{K}_{\infty}^{\beta_{2}}
$$

whenever $0 \leq \gamma_{1}<\gamma_{2}<\alpha_{0}<\beta_{1}<\beta_{2}$.

Moreover, given $\alpha \geq 0$, there exists $r>0$ such that $\mathcal{K}_{r}=\mathcal{K}_{\infty}^{\alpha}$ if and only if $\alpha_{0}<\infty$ and $\alpha>\alpha_{0}$.

Remark 4.2. In the extreme cases $\alpha_{0}=0$ and $\alpha_{0}=+\infty$, part (ii) of Theorem 4.1 is understood as follows:

(a) If $\alpha_{0}=0$, then $\mathcal{K}_{\infty}^{0} \supsetneq \mathcal{K}_{\infty}^{\beta_{1}}=\mathcal{K}_{\infty}^{\beta_{2}}$ for $0<\beta_{1}<\beta_{2}<\infty$.

(b) If $\alpha_{0}=+\infty$, then $\mathcal{K}_{\infty}^{\gamma_{1}} \supsetneq \mathcal{K}_{\infty}^{\gamma_{2}}$ for $0 \leq \gamma_{1}<\gamma_{2}<\infty$.

We remark that $\mathcal{K}_{\infty}^{\alpha}$ is not defined for $\alpha=+\infty$.

We may rephrase the statement of the theorem by saying that there are only two mutually exclusive cases: (1) the reachability spaces $\mathcal{K}_{\infty}^{\alpha}$ are all equal for all values of the parameter $\alpha \geq 0 ;(2)$ the reachability spaces $\mathcal{K}_{\infty}^{\alpha}$ coincide for $\alpha$ larger than some critical value $\alpha_{0}$, but $\mathcal{K}_{\infty}^{\alpha}$ are all distinct for $\alpha \in\left[0, \alpha_{0}\right]$ (and strictly larger than $\mathcal{K}_{\infty}^{\alpha}$, $\alpha>\alpha_{0}$ ). Moreover, in case 1 the states reachable in an unbounded time interval can also be reached in a finite time interval, independently of the value of $\alpha$. In case 2 the states in $\mathcal{K}_{\infty}^{\alpha}$ can be reached in a finite time interval if and only if $\alpha>\alpha_{0}$.

In section 5 we will give examples to show that cases (i) and (ii) of Theorem 4.1 may occur and, in case (ii), each of the possibilities $\alpha_{0}=0,0<\alpha_{0}<\infty, \alpha_{0}=\infty$ may occur.

In section 4.1 we collect some preliminary results for the proof of Theorem 4.1; the proof is presented in section 4.2. 
4.1. Some auxiliary operators. The proof of Theorem 4.1 is based on some properties of the operators

$$
S_{0}(t)=Q_{\infty}^{-1 / 2} e^{t A} Q_{\infty}^{1 / 2}, \quad t \geq 0,
$$

that we are going to study in this subsection. These operators have been introduced independently in [11] and [3]. The following result, proved in these papers, shows in particular that $S_{0}(t)$ are everywhere defined bounded linear operators on $H$, with norm less or equal to 1 . We report the proof for completeness, with some simplifications contained in [12].

Proposition 4.3. Assume Hypothesis 3.1. For every $t>0$ we have

$$
i m\left(e^{t A} Q_{\infty}^{1 / 2}\right) \subset i m Q_{\infty}^{1 / 2} \quad \text { and } \quad\left\|Q_{\infty}^{-1 / 2} e^{t A} Q_{\infty}^{1 / 2}\right\| \leq 1 .
$$

Moreover, the following conditions are equivalent:

(i) $\left\|S_{0}(t)\right\|<1$;

(ii) $i m Q_{\infty}^{1 / 2}=i m Q_{t}^{1 / 2}$.

Proof. By Lemma 2.2, in order to prove (4.3), it suffices to show

$$
\left|Q_{\infty}^{1 / 2} e^{t A^{*}} x\right|^{2} \leq\left|Q_{\infty}^{1 / 2} x\right|^{2}, \quad x \in H,
$$

i.e., $e^{t A} Q_{\infty} e^{t A^{*}} \leq Q_{\infty}$. This follows from the definition of $Q_{\infty}$, since

$$
e^{t A} Q_{\infty} e^{t A^{*}}=\int_{0}^{\infty} e^{(t+s) A} B B^{*} e^{(t+s) A^{*}} d s=\int_{t}^{\infty} e^{r A} B B^{*} e^{r A^{*}} d r=Q_{\infty}-Q_{t} \leq Q_{\infty} .
$$

Equation (4.3) is now proved.

First note that $Q_{t} \leq Q_{\infty}$, so by Lemma 2.2 the inclusion im $Q_{t}^{1 / 2} \subset i m Q_{\infty}^{1 / 2}$ always holds. By Lemma 2.2 again, (i) holds if and only if there exists $\alpha \in(0,1)$ such that

$$
\left|Q_{\infty}^{1 / 2} e^{t A^{*}} x\right|^{2} \leq \alpha\left|Q_{\infty}^{1 / 2} x\right|^{2}, \quad x \in H .
$$

Now note that (4.4) implies

$$
\left|Q_{\infty}^{1 / 2} e^{t A^{*}} x\right|^{2}=\left\langle\left(Q_{\infty}-Q_{t}\right) x, x\right\rangle
$$

so (i) holds if and only if

$$
\left\langle\left(Q_{\infty}-Q_{t}\right) x, x\right\rangle \leq \alpha\left\langle Q_{\infty} x, x\right\rangle, \quad x \in H,
$$

or

$$
\left\langle Q_{\infty} x, x\right\rangle \leq(1-\alpha)^{-1}\left\langle Q_{t} x, x\right\rangle, \quad x \in H .
$$

By Lemma 2.2 this is equivalent to $i m Q_{\infty}^{1 / 2} \subset i m Q_{t}^{1 / 2}$.

The following proposition is the main step toward the proof of Theorem 4.1.

Proposition 4.4. Assume Hypothesis 3.1.

(i) If there exists $r>0$ such that $\left\|S_{0}(r)\right\|<1$, then for every $\alpha>0$ there exists $c>0$ such that $c Q_{\infty} \leq Q_{\infty}^{(\alpha)}$.

(ii) If there exist $c>0$ and $\alpha>0$ such that $c Q_{\infty} \leq Q_{\infty}^{(\alpha)}$, then there exists $r>0$ such that $\left\|S_{0}(r)\right\|<1$. 
Proof. We first note the identity, for $h \in H$ and $r>0$,

$$
\int_{0}^{r}\left|B^{*} e^{t A^{*}} h\right|^{2} d t=\left|Q_{\infty}^{1 / 2} h\right|^{2}-\left|Q_{\infty}^{1 / 2} e^{r A^{*}} h\right|^{2},
$$

which follows from the computation

$$
\begin{aligned}
\int_{0}^{r}\left|B^{*} e^{t A^{*}} h\right|^{2} d t & =\int_{0}^{\infty}\left|B^{*} e^{t A^{*}} h\right|^{2} d t-\int_{r}^{\infty}\left|B^{*} e^{t A^{*}} h\right|^{2} d t \\
& =\left|Q_{\infty}^{1 / 2} h\right|^{2}-\int_{0}^{\infty}\left|B^{*} e^{t A^{*}} e^{r A^{*}} h\right|^{2} d t \\
& =\left|Q_{\infty}^{1 / 2} h\right|^{2}-\left|Q_{\infty}^{1 / 2} e^{r A^{*}} h\right|^{2} .
\end{aligned}
$$

Let us prove (i). We have

$$
\left\langle Q_{\infty}^{(\alpha)} h, h\right\rangle=\int_{0}^{\infty} e^{-2 \alpha t}\left|B^{*} e^{t A^{*}} h\right|^{2} d t \geq e^{-2 \alpha r} \int_{0}^{r}\left|B^{*} e^{t A^{*}} h\right|^{2} d t,
$$

and using (4.5) we obtain

$$
\left|\left(Q_{\infty}^{(\alpha)}\right)^{1 / 2} h\right|^{2} \geq e^{-2 \alpha r}\left(\left|Q_{\infty}^{1 / 2} h\right|^{2}-\left|Q_{\infty}^{1 / 2} e^{r A^{*}} h\right|^{2}\right) .
$$

Since $Q_{\infty}^{1 / 2} e^{r A^{*}} h=S_{0}(r)^{*} Q_{\infty}^{1 / 2} h$, we arrive at

$$
\left|\left(Q_{\infty}^{(\alpha)}\right)^{1 / 2} h\right|^{2} \geq e^{-2 \alpha r}\left(1-\left\|S_{0}(r)\right\|^{2}\right)\left|Q_{\infty}^{1 / 2} h\right|^{2},
$$

and the conclusion follows with $c=e^{-2 \alpha r}\left(1-\left\|S_{0}(r)\right\|^{2}\right)$.

Now we prove (ii). We can assume $c<1$. We have

$$
\begin{aligned}
& \left|Q_{\infty}^{1 / 2} e^{r A^{*}} h\right|^{2}=\int_{0}^{\infty}\left|B^{*} e^{t A^{*}} e^{r A^{*}} h\right|^{2} d t=\int_{r}^{\infty}\left|B^{*} e^{t A^{*}} h\right|^{2} d t \geq e^{2 r \alpha} \int_{r}^{\infty} e^{-2 t \alpha}\left|B^{*} e^{t A^{*}} h\right|^{2} d t \\
& \quad=e^{2 r \alpha} \int_{0}^{\infty} e^{-2 t \alpha}\left|B^{*} e^{t A^{*}} h\right|^{2} d t-e^{2 r \alpha} \int_{0}^{r} e^{-2 t \alpha}\left|B^{*} e^{t A^{*}} h\right|^{2} d t \\
& \quad=e^{2 r \alpha}\left|\left(Q_{\infty}^{(\alpha)}\right)^{1 / 2} h\right|^{2}-e^{2 r \alpha} \int_{0}^{r} e^{-2 t \alpha}\left|B^{*} e^{t A^{*}} h\right|^{2} d t \\
& \quad \geq e^{2 r \alpha}\left|\left(Q_{\infty}^{(\alpha)}\right)^{1 / 2} h\right|^{2}-e^{2 r \alpha} \int_{0}^{r}\left|B^{*} e^{t A^{*}} h\right|^{2} d t .
\end{aligned}
$$

By assumption we have $\left|\left(Q_{\infty}^{(\alpha)}\right)^{1 / 2} h\right|^{2} \geq c\left|Q_{\infty}^{1 / 2} h\right|^{2}$, and using (4.5) we obtain

$$
\left|Q_{\infty}^{1 / 2} e^{r A^{*}} h\right|^{2} \geq c e^{2 r \alpha}\left|Q_{\infty}^{1 / 2} h\right|^{2}-e^{2 r \alpha}\left(\left|Q_{\infty}^{1 / 2} h\right|^{2}-\left|Q_{\infty}^{1 / 2} e^{r A^{*}} h\right|^{2}\right)
$$

or

$$
\left|Q_{\infty}^{1 / 2} e^{r A^{*}} h\right|^{2} \leq(1-c) e^{2 r \alpha}\left(e^{2 r \alpha}-1\right)^{-1}\left|Q_{\infty}^{1 / 2} h\right|^{2} .
$$

Choosing $r>0$ so large that $\gamma:=(1-c) e^{2 r \alpha}\left(e^{2 r \alpha}-1\right)^{-1}<1$, and noting again that $Q_{\infty}^{1 / 2} e^{r A^{*}} h=S_{0}(r)^{*} Q_{\infty}^{1 / 2} h$, we obtain

$$
\left|S_{0}(r)^{*} Q_{\infty}^{1 / 2} h\right|^{2} \leq \gamma\left|Q_{\infty}^{1 / 2} h\right|^{2}, \quad h \in H .
$$

This proves that $\left|S_{0}(r)^{*} k\right|^{2} \leq \gamma|k|^{2}$ for $k \in i m Q_{\infty}^{1 / 2}$. Since, however, by its definition, $S_{0}(r) k=0$ for $k$ in the kernel of $Q_{\infty}^{1 / 2}$, which is the orthogonal subspace to $i m Q_{\infty}^{1 / 2}$, 
it follows that $\left|S_{0}(r)^{*} k\right|^{2} \leq \gamma|k|^{2}$ for every $k \in H$, which proves that $\left\|S_{0}(r)\right\|^{2} \leq$ $\gamma<1$.

Replacing $A$ with $A-\alpha I$ in the definition of $S_{0}(t)$, we get a different family of operators that we will denote $S_{0}^{(\alpha)}(t)$, namely,

$$
S_{0}^{(\alpha)}(t)=e^{-\alpha t}\left(Q_{\infty}^{(\alpha)}\right)^{-1 / 2} e^{t A}\left(Q_{\infty}^{(\alpha)}\right)^{1 / 2}, \quad t \geq 0, \alpha \geq 0 .
$$

Note that $S_{0}^{(0)}(t)=S_{0}(t)$. As an immediate consequence of the properties enjoyed by $S_{0}(t)$, we obtain the following result.

Corollary 4.5. Assume Hypothesis 3.1 and let $\alpha \geq 0$.

(i) If there exists $r>0$ such that $\left\|S_{0}^{(\alpha)}(r)\right\|<1$, then for every $\beta>\alpha$ there exists $c>0$ such that $c Q_{\infty}^{(\alpha)} \leq Q_{\infty}^{(\beta)}$.

(ii) If there exist $c>0$ and $\beta>\alpha$ such that $c Q_{\infty}^{(\alpha)} \leq Q_{\infty}^{(\beta)}$, then there exists $r>0$ such that $\left\|S_{0}^{(\alpha)}(r)\right\|<1$.

(iii) If there exist $\alpha \geq 0$ and $r>0$ such that $\left\|S_{0}^{(\alpha)}(r)\right\|<1$, then for every $\beta>\alpha$ there exists $s>0$ such that $\left\|S_{0}^{(\beta)}(s)\right\|<1$.

Proof. To prove points (i) and (ii) it suffices to apply Proposition 4.4, replacing $A$ and $\alpha$ with $A-\alpha I$ and $\beta-\alpha$, respectively.

To prove point (iii) take $\beta_{1}>\beta$. Then by point (i) (with $\beta_{1}$ instead of $\beta$ ) there exists $c>0$ such that $c Q_{\infty}^{(\alpha)} \leq Q_{\infty}^{\left(\beta_{1}\right)}$. By point (ii) (with $\beta$ instead of $\alpha$ ) there exists $s>0$ such that $\left\|S_{0}^{(\beta)}(s)\right\|<1$.

Lemma 4.6. Assume Hypothesis 3.1 and suppose that $\left\|S_{0}^{(\alpha)}(r)\right\|<1$ for some $\alpha>0$ and $r>0$. Then for all $\gamma \in(0, \alpha)$ sufficiently close to $\alpha$ we have $\left\|S_{0}^{(\gamma)}(r)\right\|<1$.

Proof. We claim that if $\gamma \in(0, \alpha)$ is sufficiently close to $\alpha$, then there exists $c>0$ such that

$$
Q_{\infty}^{(\gamma)} \leq c Q_{\infty}^{(\alpha)}
$$

Assume the claim for a moment. Since the opposite inequality $Q_{\infty}^{(\gamma)} \geq Q_{\infty}^{(\alpha)}$ is obvious, we conclude by Lemma 2.2 that $i m\left(Q_{\infty}^{(\alpha)}\right)^{1 / 2}=i m\left(Q_{\infty}^{(\gamma)}\right)^{1 / 2}$. Since we assume $\left\|S_{0}^{(\alpha)}(r)\right\|<1$, we can apply Proposition 4.3 (replacing $A$ with $A-\alpha I$ ) and conclude that $i m\left(Q_{\infty}^{(\alpha)}\right)^{1 / 2}=i m\left(Q_{r}^{(\alpha)}\right)^{1 / 2}$. Since the latter space is clearly identical to $i m\left(Q_{r}^{(\gamma)}\right)^{1 / 2}$, we also have $i m\left(Q_{r}^{(\gamma)}\right)^{1 / 2}=i m\left(Q_{\infty}^{(\gamma)}\right)^{1 / 2}$, and applying Proposition 4.3 again (replacing $A$ with $A-\gamma I$ ) we conclude that $\left\|S_{0}^{(\gamma)}(r)\right\|<1$, and the lemma is proved.

It remains to prove (4.7). For $0 \leq \gamma<\alpha$ and $h \in H$,

$$
\left\langle e^{t A} Q_{\infty}^{(\alpha)} e^{t A^{*}} h, h\right\rangle=\int_{0}^{\infty}\left|B^{*} e^{(t+s) A^{*}} h\right|^{2} e^{-2 \alpha s} d s=e^{2 \alpha t} \int_{t}^{\infty}\left|B^{*} e^{s A^{*}} h\right|^{2} e^{-2 \alpha s} d s .
$$

Integrating by parts, we have, for $T>0$,

$$
\begin{aligned}
& \int_{0}^{T} e^{-2 \gamma t}\left\langle e^{t A} Q_{\infty}^{(\alpha)} e^{t A^{*}} h, h\right\rangle d t \\
& =\int_{0}^{T} e^{2(\alpha-\gamma) t} \int_{t}^{\infty}\left|B^{*} e^{s A^{*}} h\right|^{2} e^{-2 \alpha s} d s d t \\
& =\frac{e^{2(\alpha-\gamma) T}}{2(\alpha-\gamma)} \int_{T}^{\infty}\left|B^{*} e^{s A^{*}} h\right|^{2} e^{-2 \alpha s} d s-\frac{1}{2(\alpha-\gamma)}\left\langle Q_{\infty}^{(\alpha)} h, h\right\rangle \\
& +\frac{1}{2(\alpha-\gamma)} \int_{0}^{T} e^{-2 \gamma t}\left|B^{*} e^{t A^{*}} h\right|^{2} d t .
\end{aligned}
$$


Since

$$
e^{2(\alpha-\gamma) T} \int_{T}^{\infty}\left|B^{*} e^{s A^{*}} h\right|^{2} e^{-2 \alpha s} d s \leq \int_{T}^{\infty}\left|B^{*} e^{s A^{*}} h\right|^{2} e^{-2 \gamma s} d s \rightarrow 0
$$

as $T \rightarrow \infty$, we arrive at the identity

$$
2(\alpha-\gamma) \int_{0}^{\infty} e^{-2 \gamma t}\left\langle e^{t A} Q_{\infty}^{(\alpha)} e^{t A^{*}} h, h\right\rangle d t=\left\langle Q_{\infty}^{(\gamma)} h, h\right\rangle-\left\langle Q_{\infty}^{(\alpha)} h, h\right\rangle .
$$

We note that $\left\langle e^{t A} Q_{\infty}^{(\alpha)} e^{t A^{*}} h, h\right\rangle=e^{2 \alpha t}\left\langle\left(Q_{\infty}^{(\alpha)}\right)^{1 / 2} S_{0}^{(\alpha)}(t) S_{0}^{(\alpha)}(t)^{*}\left(Q_{\infty}^{(\alpha)}\right)^{1 / 2} h, h\right\rangle$, so if we define

$$
R h=2(\alpha-\gamma) \int_{0}^{\infty} e^{2(\alpha-\gamma) t} S_{0}^{(\alpha)}(t) S_{0}^{(\alpha)}(t)^{*} h d t, \quad h \in H
$$

and we assume for a moment that $R$ is a well-defined bounded linear operator, we conclude that

$$
\left\langle\left(Q_{\infty}^{(\alpha)}\right)^{1 / 2} R\left(Q_{\infty}^{(\alpha)}\right)^{1 / 2} h, h\right\rangle=\left\langle Q_{\infty}^{(\gamma)} h, h\right\rangle-\left\langle Q_{\infty}^{(\alpha)} h, h\right\rangle,
$$

and consequently $Q_{\infty}^{(\gamma)} \leq(1+\|R\|) Q_{\infty}^{(\alpha)}$, which proves (4.7). To show that $R$ is well defined, we first note that the family $\left\{S_{0}^{(\alpha)}(t), t \geq 0\right\}$ is a contraction semigroup, and since we assume that $\left\|S_{0}^{(\alpha)}(r)\right\|<1$ for some $r>0$, it follows easily that there exist $M, \omega>0$ such that $\left\|S_{0}^{(\alpha)}(t)\right\| \leq M e^{-\omega t}$ for every $t>0$. If $\alpha-\gamma<\omega$, then the integral defining $R$ is convergent (as a Bochner integral). This finishes the proof.

4.2. Proof of Theorem 4.1. During the proof we repeatedly use the following properties proved above: for $0 \leq \alpha<\beta$ and $r>0$

$$
\mathcal{K}_{\infty}^{\alpha}=i m\left(Q_{\infty}^{(\alpha)}\right)^{1 / 2}, \quad \mathcal{K}_{r}=i m Q_{r}^{1 / 2}, \quad \mathcal{K}_{\infty}^{\alpha} \supset \mathcal{K}_{\infty}^{\beta} \supset \mathcal{K}_{r} .
$$

We consider two mutually exclusive cases (i) and (ii).

Case (i). Suppose that we have $\left\|S_{0}(r)\right\|<1$ for some $r>0$.

By Proposition 4.4 (i) and Lemma 2.2 we have $i m Q_{\infty}^{1 / 2} \subset i m\left(Q_{\infty}^{(\alpha)}\right)^{1 / 2}$ for all $\alpha \geq 0$ or, equivalently, $\mathcal{K}_{\infty} \subset \mathcal{K}_{\infty}^{\alpha}$ so that in fact $\mathcal{K}_{\infty}=\mathcal{K}_{\infty}^{\alpha}$ for all $\alpha \geq 0$. Applying Proposition 4.3, we also have $\mathcal{K}_{\infty}=i m Q_{\infty}^{1 / 2}=i m Q_{r}^{1 / 2}=\mathcal{K}_{r}$.

Case (ii). Suppose $\left\|S_{0}(r)\right\|=1$ for every $r>0$.

We define

$$
J=\left\{\alpha \geq 0:\left\|S_{0}^{(\alpha)}(r)\right\|=1 \text { for every } r>0\right\}, \quad \alpha_{0}=\sup J .
$$

Note that the set $J$ contains at least $\alpha=0$, so $\alpha_{0}$ is well defined and $0 \leq \alpha_{0} \leq \infty$. By Corollary 4.5 (iii) only the following cases can occur:

(a) $\alpha_{0}=0, J=\{0\}$;

(b) $0<\alpha_{0}<\infty, J=\left[0, \alpha_{0}\right]$;

(c) $\alpha_{0}=\infty, J=[0, \infty)$.

Note that the case $0<\alpha_{0}<\infty, J=\left[0, \alpha_{0}\right)$ is impossible by Lemma 4.6.

Suppose $0 \leq \gamma_{1}<\gamma_{2}<\alpha_{0} \leq \infty$. Assume by contradiction that $\mathcal{K}_{\infty}^{\gamma_{1}}=\mathcal{K}_{\infty}^{\gamma_{2}}$ (respectively, that $\alpha_{0}<\infty$ and $\mathcal{K}_{\infty}^{\gamma_{2}}=\mathcal{K}_{\infty}^{\alpha_{0}}$ ). Then by (4.8) and Lemma 2.2 we have $c Q_{\infty}^{\left(\gamma_{1}\right)} \leq Q_{\infty}^{\left(\gamma_{2}\right)}$ (respectively, $c Q_{\infty}^{\left(\gamma_{2}\right)} \leq Q_{\infty}^{\left(\alpha_{0}\right)}$ ) for some $c>0$, and Corollary 4.5 (ii) 
implies that there exists $r>0$ such that $\left\|S_{0}^{\left(\gamma_{1}\right)}(r)\right\|<1$ (respectively, $\left\|S_{0}^{\left(\gamma_{2}\right)}(r)\right\|<$ 1 ), which contradicts the definition of $\alpha_{0}$. Next note that the equality $\mathcal{K}_{\infty}^{\gamma_{2}}=\mathcal{K}_{r}$ (respectively, $\alpha_{0}<\infty$ and $\mathcal{K}_{\infty}^{\alpha_{0}}=\mathcal{K}_{r}$ ) cannot hold for any $r>0$ since by Proposition 4.3, applied to $A-\gamma_{2} I$ instead of $A$ (respectively, applied to $A-\alpha_{0} I$ instead of $A$ ), it would imply that $\left\|S_{0}^{\left(\gamma_{2}\right)}(r)\right\|<1$ (respectively, $\left\|S_{0}^{\left(\alpha_{0}\right)}(r)\right\|<1$ ), which is impossible.

Now suppose that $0 \leq \alpha_{0}<\beta_{1}<\beta_{2}<\infty$. Assume by contradiction that $\mathcal{K}_{\infty}^{\alpha_{0}}=\mathcal{K}_{\infty}^{\beta_{1}}$. Then by (4.8) and Lemma 2.2 we have $c Q_{\infty}^{\left(\alpha_{0}\right)} \leq Q_{\infty}^{\left(\beta_{1}\right)}$, and Corollary 4.5 (ii) implies that there exists $r>0$ such that $\left\|S_{0}^{\left(\alpha_{0}\right)}(r)\right\|<1$, which is impossible. Next note that by the definition of $\alpha_{0}$ there exists $r>0$ such that $\left\|S_{0}^{\left(\beta_{1}\right)}(r)\right\|<1$. By Corollary 4.5 (i) and Lemma 2.2 we have $i m\left(Q_{\infty}^{\left(\beta_{1}\right)}\right)^{1 / 2} \subset i m\left(Q_{\infty}^{\left(\beta_{2}\right)}\right)^{1 / 2}$ or, equivalently, $\mathcal{K}_{\infty}^{\beta_{1}} \subset \mathcal{K}_{\infty}^{\beta_{2}}$ so that in fact $\mathcal{K}_{\infty}^{\beta_{1}}=\mathcal{K}_{\infty}^{\beta_{2}}$. Finally, applying Proposition 4.3 (with $A-\beta_{1} I$ instead of $A$ ), we also have $\mathcal{K}_{\infty}^{\beta_{1}}=i m\left(Q_{\infty}^{\left(\beta_{1}\right)}\right)^{1 / 2}=i m Q_{r}^{1 / 2}=\mathcal{K}_{r}$.

Theorem 4.1 is now completely proved.

This proof also describes a criterion to decide which case in Theorem 4.1 occurs, as well as the value of $\alpha_{0}$.

Corollary 4.7. Assume Hypothesis 3.1. Let $Q_{\infty}^{(\alpha)}$ and $S_{0}^{(\alpha)}$ be defined by (4.2) and (4.6), respectively. Case (ii) in Theorem 4.1 occurs if and only if the set

$$
J=\left\{\alpha \geq 0:\left\|S_{0}^{(\alpha)}(r)\right\|=1 \text { for every } r>0\right\}
$$

is nonempty, and then $\alpha_{0}=\sup J \in[0, \infty]$.

4.3. Approximately reachable states. If the concept of reachability used so far is replaced by approximate reachability, then the behavior of the perturbed system (4.1) is completely different. This is a well-known fact, but we prefer to report a direct proof in this short section since it is often used in a rather implicit way and it is not easy to find a reference (see, however, Chapter 9 of [19]).

First, let us consider the control system (3.2) and define $\mathcal{H}_{r}$, the space of approximately reachable states from zero in time $r$, as the set of all elements $k \in H$ such that for all $\epsilon>0$ there exists a control $u \in L^{2}(T-r, T ; U)$ such that $|y(T)-k|<\epsilon$, where $y$ denotes the corresponding trajectory of system (3.2) with $h=0$.

In an analogous way we define the space $\mathcal{H}_{\infty}$ as the space of approximately reachable states over the unbounded time interval $(-\infty, T]$ for system (3.3), corresponding to controls $u \in L^{2}(-\infty, T ; U)$.

For the perturbed system

$$
y_{\alpha}^{\prime}(t)=(A-\alpha I) y_{\alpha}(t)+B u(t),
$$

with $\alpha \geq 0$, the spaces of approximately reachable states will be denoted by $\mathcal{H}_{r}^{\alpha}$ and $\mathcal{H}_{\infty}^{\alpha}$, respectively, for the case of a bounded time interval and an unbounded one. Clearly, $\mathcal{H}_{r}^{\alpha}=\mathcal{H}_{r}$.

We claim that $\mathcal{H}_{\infty}^{\alpha}=\cup_{r} \mathcal{H}_{r}$ for all $\alpha$, so, in particular, $\mathcal{H}_{\infty}^{\alpha}$ is independent of $\alpha$.

To prove the claim, let us take an element $k \in \mathcal{H}_{\infty}^{\alpha}$; we want to prove that there exists a suitable $r$ such that $k \in \mathcal{H}_{r}^{\alpha}=\mathcal{H}_{r}$. In fact, by definition of the trajectory of a control system (compare with formula (3.4)) and by assumption (3.1), we have that for all $\epsilon>0$ there exists $r>0$ so large that $\left|y_{\alpha}(T)-\tilde{y}_{\alpha}(T)\right|<\epsilon$, where $\tilde{y}_{\alpha}$ denotes the trajectory of the system (4.9), starting from zero at time $T-r$, driven by the control $\tilde{u} \in L^{2}(T-r, T ; U)$ which coincides with $u$ on $[T-r, T]$. By triangular inequality we conclude that $k \in \mathcal{H}_{r}^{\alpha}$, and the claim is proved. 
5. Examples and remarks. The aim of this section is to show that all the situations in the statement of Theorem 4.1 may occur, namely, case (i) or case (ii) with $\alpha_{0}=0$ or $0<\alpha_{0}<\infty$ or $\alpha_{0}=\infty$. The value of $\alpha_{0}$ is difficult to compute in general, since it is defined in terms of the semigroup $S_{0}^{(\alpha)}$ (compare with Corollary 4.7) and not in terms of $A$ and $B$. Nevertheless, in some interesting cases we do have explicit solutions to the problem. We also discuss the relevance of Theorem 4.1 to special classes of systems - for instance, the finite-dimensional systems or the null controllable ones.

\subsection{Case (i) of Theorem 4.1.}

\subsubsection{The finite-dimensional case.}

Corollary 5.1. Assume that Hypothesis 3.1 holds and that $\operatorname{dim} H<\infty$. Then case (i) of Theorem 4.1 occurs and $\mathcal{K}_{\infty}^{\alpha}=\mathcal{K}_{t}$ for every $t>0$ and $\alpha \geq 0$.

Thus every state reachable in an unbounded time interval (no matter what the value of $\alpha$ is) can be reached in an arbitrarily small time $t>0$.

Proof. We claim that

$$
i m Q_{\infty}^{1 / 2}=i m Q_{t}^{1 / 2}, \quad t>0 .
$$

Since $i m Q_{\infty}^{1 / 2}=\mathcal{K}_{\infty}$, case (i) of Theorem 4.1 occurs, and the reachability spaces $\mathcal{K}_{\infty}^{\alpha}$ coincide for all $\alpha \geq 0$ and in fact they coincide with $\mathcal{K}_{t}$ for every $t>0$.

To prove the claim we note that if $\operatorname{dim} H<\infty$, then (5.1) is equivalent to $\operatorname{ker} Q_{\infty}^{1 / 2}=\operatorname{ker} Q_{t}^{1 / 2}$ (ker denotes of course the kernel of an operator). Clearly, $\operatorname{ker} Q_{\infty}^{1 / 2} \subset \operatorname{ker} Q_{t}^{1 / 2}$. Conversely, if $Q_{t}^{1 / 2} h=0$ for some $h \in H$, then $Q_{t} h=0$ and $Q^{1 / 2} e^{s A} h=0$ for every $s \in[0, t]$. By analyticity, $Q^{1 / 2} e^{s A} h=0$ for every $s \geq 0$. This implies $Q_{\infty} h=0$ and consequently $Q_{\infty}^{1 / 2} h=0$.

\subsubsection{Exactly null controllable systems.}

Corollary 5.2. Assume that Hypothesis 3.1 holds and that there exists $r>0$ such that

$$
i m e^{r A} \subset i m Q_{r}^{1 / 2} \text {. }
$$

Then case (i) of Theorem 4.1 occurs and in fact $\mathcal{K}_{\infty}^{\alpha}=\mathcal{K}_{r}$ for every $\alpha \geq 0$.

It is well known that (5.2) is equivalent to the exact null controllability property in time $r$, defined as follows: for every $h \in H$ there exists a control $u \in L^{2}([T-r, T] ; U)$ such that the trajectory of the control system

$$
y^{\prime}(t)=A y(t)+B u(t), \quad t \in[T-r, T], \quad y(T-r)=h,
$$

satisfies $y(T)=0$ (see, e.g., [7], [21]). The pair $(A, B)$ is then called a null controllable pair (in time $r$ ). It is also well known that this property holds if $B=I$ (for every $r>0)$.

Proof. We claim that (5.2) implies

$$
i m Q_{\infty}^{1 / 2}=i m Q_{r}^{1 / 2} .
$$

Then it follows from Proposition 4.3 that $\left\|S_{0}(r)\right\|<1$ and by Corollary 4.7 we conclude that case (i) in Theorem 4.1 occurs.

The claim is proved in [8, Theorem 11.13], but we nevertheless include the following simpler proof. First note that the inequality $Q_{r} \leq Q_{\infty}$ implies that $i m Q_{r}^{1 / 2} \subset$ 
im $Q_{\infty}^{1 / 2}$ by Lemma 2.2. Next note that the inclusion (5.2) implies that the operator $\Gamma_{r}:=Q_{r}^{-1 / 2} e^{r A}$ is everywhere defined, and since it is closed, it is also bounded, by the closed graph theorem. From the definition of $Q_{\infty}$ it is easy to obtain the identity $e^{r A} Q_{\infty} e^{r A^{*}}+Q_{r}=Q_{\infty}$, and it follows that $Q_{r}^{1 / 2}\left(\Gamma_{r} Q_{\infty} \Gamma_{r}^{*}+I\right) Q_{r}^{1 / 2}=Q_{\infty}$, which implies $\left|Q_{\infty}^{1 / 2} x\right|^{2} \leq\left(1+\left\|\Gamma_{r} Q_{\infty} \Gamma_{r}^{*}\right\|\right)\left|Q_{r}^{1 / 2} x\right|^{2}, x \in H$, and so, by Lemma 2.2, $i m Q_{\infty}^{1 / 2} \subset i m Q_{r}^{1 / 2}$, and (5.3) is proved.

\subsubsection{A special case.}

Corollary 5.3. Suppose that Hypothesis 3.1 holds and that, setting $Q=B B^{*}$, we have

$$
i m e^{t A} Q^{1 / 2} \subset i m Q^{1 / 2} \quad \text { and } \quad\left\|Q^{-1 / 2} e^{t A} Q^{1 / 2}\right\| \leq M e^{-\beta t}, \quad t \geq 0,
$$

for some constants $\beta>0, M>0$. Then case (i) of Theorem 4.1 occurs and $\mathcal{K}_{\infty}^{\alpha}=\mathcal{K}_{r}$ for every $\alpha \geq 0$ and $r>0$.

We remark that $i m B=i m Q^{1 / 2}$ by Lemma 2.2. Moreover, the inclusion in (5.4) implies that the linear operators $Q^{-1 / 2} e^{t A} Q^{1 / 2}$ are everywhere defined and therefore, being obviously closed, they are also continuous.

We note that all the assumptions of the corollary hold true if $B$ is a bounded linear operator from $U$ to $H$ and $A$ is the infinitesimal generator of an exponentially stable, strongly continuous semigroup of operators that commute with $Q=B B^{*}$. Thus there are many control systems satisfying these assumptions.

Proof. We define $\widehat{S}(t)=Q^{-1 / 2} e^{t A} Q^{1 / 2}$ for $t>0, \widehat{S}(0)=I$. Clearly, $\widehat{S}$ is a semigroup of bounded linear operators on $H$, satisfying $\|\widehat{S}(t)\| \leq M e^{-\beta t}, t \geq 0$. We note that for $x \in H, y \in i m Q^{1 / 2}$,

$$
\langle\widehat{S}(t) x, y\rangle=\left\langle e^{t A} Q^{1 / 2} x, Q^{-1 / 2} y\right\rangle \rightarrow\langle x, y\rangle, \quad \text { as } t \rightarrow 0 .
$$

Since $\widehat{S}$ is bounded in the operator norm, it follows that (5.5) holds for every $x \in H$ and $y \in \overline{i m Q^{1 / 2}}$ (the closure of $i m Q^{1 / 2}$ in $H$ ). Since $\widehat{S}(t) x=Q^{-1 / 2} e^{t A} Q^{1 / 2} x$ is orthogonal to $\operatorname{ker} Q^{1 / 2}=\overline{i m Q^{1 / 2}},(5.5)$ holds for all $x, y \in H$. Thus, for every $x \in H, \widehat{S}(t) x \rightarrow x$ weakly in $H$; it is well known that this implies that $\widehat{S}$ is a strongly continuous semigroup.

Let $\widehat{A}$ denote the infinitesimal generator of $\widehat{S}$. We consider the pair $(\widehat{A}, I)$, and we define the corresponding controllability operators

$$
\widehat{Q}_{\infty} x=\int_{0}^{\infty} \widehat{S}(t) \widehat{S}(t)^{*} x d t, \quad \widehat{Q}_{r} x=\int_{0}^{r} \widehat{S}(t) \widehat{S}(t)^{*} x d t, \quad x \in H, r>0 .
$$

Since the pair $(\widehat{A}, I)$ is null controllable in time $r$ for every $r>0$, by the results of the previous paragraph we conclude that there exists a constant $C_{r}>0$ such that $\left|\widehat{Q}_{\infty}^{1 / 2} x\right|^{2} \leq C_{r}\left|\widehat{Q}_{r}^{1 / 2} x\right|^{2}$. Since $Q^{1 / 2} \widehat{S}(t)=e^{t A} Q^{1 / 2}$, it follows that

$Q^{1 / 2} \widehat{Q}_{\infty} Q^{1 / 2} x=\int_{0}^{\infty} Q^{1 / 2} \widehat{S}(t) \widehat{S}(t)^{*} Q^{1 / 2} x d t=\int_{0}^{\infty} e^{t A} Q e^{t A^{*}} x d t=Q_{\infty} x, \quad x \in H$,

and, similarly, $Q^{1 / 2} \widehat{Q}_{r} Q^{1 / 2}=Q_{r}$. Therefore,

$$
\left|Q_{\infty}^{1 / 2} x\right|^{2}=\left|\widehat{Q}_{\infty}^{1 / 2} Q^{1 / 2} x\right|^{2} \leq C_{r}\left|\widehat{Q}_{r}^{1 / 2} Q^{1 / 2} x\right|^{2}=C_{r}\left|Q_{r}^{1 / 2} x\right|^{2}, \quad x \in H,
$$

which implies $i m Q_{\infty}^{1 / 2} \subset i m Q_{r}^{1 / 2}$. Since the opposite inclusion is obvious, we have $i m Q_{\infty}^{1 / 2}=i m Q_{r}^{1 / 2}$ for $r>0$ and the result follows. 


\subsubsection{Exactly reachable systems.}

Corollary 5.4. Suppose that Hypothesis 3.1 holds and that $A$ is exponentially stable. If $\mathcal{K}_{\infty}=H$, then case (i) of Theorem 4.1 occurs and consequently $\mathcal{K}_{r}=H$ for $r>0$ sufficiently large.

Systems satifying the condition $\mathcal{K}_{\infty}=H$ may be called exactly reachable on $(-\infty, T]$. Thus, if $A$ is exponentially stable, exact reachability on $(-\infty, T]$ implies exact reachability on a bounded interval $[T-r, T]$.

Proof. By a standard duality argument one can check that the equality $\mathcal{K}_{\infty}=H$ is equivalent to the following condition: there exists $\kappa>0$ such that

$$
\int_{0}^{\infty}\left|B^{*} e^{t A^{*}} x\right|^{2} d t \geq \kappa|x|^{2}, \quad x \in H
$$

Condition (5.6) is called exact observability for the pair $\left(A^{*}, B^{*}\right)$. Since $A$ is exponentially stable, it follows from Proposition 2.8 in [18] that (5.6) holds if and only if there exist $r>0$ and $\kappa_{r}>0$ such that

$$
\int_{0}^{r}\left|B^{*} e^{t A^{*}} x\right|^{2} d t \geq \kappa_{r}|x|^{2}, \quad x \in H
$$

and this is equivalent to the equality $\mathcal{K}_{r}=H$, again by duality.

5.2. Case (ii) of Theorem 4.1 with $\alpha_{0}=0$. The example in this section was invented by Gołdys for a different purpose [14]. Let $H=U$ be a Hilbert space and $\left\{h_{k}, k \geq 1\right\}$ an orthonormal basis of $H$. Define the operators $A$ and $B$ setting

$$
A h_{k}=-\frac{1}{k} h_{k}, \quad B h_{k}=\frac{1}{k^{3 / 2}} h_{k} .
$$

Note that $A$ and $B$ are commuting bounded self-adjoint operators, and $A$ is nonpositive, but, in contrast to section 5.1.3, $A$ does not have bounded inverse. Hypothesis 3.1 is easy to verify. We have $S_{0}(t)=e^{t A}$ and $e^{t A} h_{k}=e^{-t / k} h_{k}, k \geq 1$, so that $\left\|S_{0}(t)\right\|=1$ for every $t>0$, whereas $\left\|S_{0}^{(\alpha)}(t)\right\|=e^{-\alpha t}\left\|S_{0}(t)\right\|<1$ for every $t>0$. Corollary 4.7 shows that in this example we have $\alpha_{0}=0$.

Remark 5.5. In this example the semigroup $\left(e^{t A}\right)$ is not exponentially stable. In Remark 5.6 below we will give another example, where $\alpha_{0}=0$ and $\left(e^{t A}\right)$ is exponentially stable.

5.3. Case (ii) of Theorem 4.1 with $0<\boldsymbol{\alpha}_{\mathbf{0}}<\infty$. We take $H=U=L^{2}(\mathbb{R})$. Let the operators $e^{t A}$ be the shift operators

$$
e^{t A} f(x)=f(x-t), \quad t \geq 0, x \in \mathbb{R},
$$

and let the operator $B$ be multiplication by the function $e^{-|x|}: B f(x)=e^{-|x|} f(x)$, $x \in \mathbb{R}$. Then one finds with simple calculations

$$
e^{t A^{*}} f(x)=f(x+t), \quad e^{t A} B B^{*} e^{t A^{*}} f(x)=e^{-2|x-t|} f(x), \quad t \geq 0, x \in \mathbb{R},
$$

so that $Q_{\infty}^{(\alpha)}, \alpha \geq 0$, is the multiplication operator by the function $g_{\alpha}$ :

$$
Q_{\infty}^{(\alpha)} f(x)=g_{\alpha}(x) f(x), \quad g_{\alpha}(x)=\int_{0}^{\infty} e^{-2 \alpha t} e^{-2|x-t|} d t, \quad x \in \mathbb{R} .
$$


It is immediate to verify (3.1), and so Hypothesis 3.1 holds. Elementary computations show that for $\alpha \neq 1$

$$
g_{\alpha}(x)= \begin{cases}\frac{e^{-2 x}}{2(\alpha-1)}\left(1-\frac{2}{\alpha+1} e^{-2(\alpha-1) x}\right) & \text { for } \quad x>0, \\ \frac{e^{2 x}}{2(\alpha+1)} & \text { for } \quad x \leq 0\end{cases}
$$

whereas

$$
g_{1}(x)= \begin{cases}e^{-2 x}\left(x+\frac{1}{4}\right) & \text { for } \quad x>0 \\ \frac{e^{2 x}}{4} & \text { for } \quad x \leq 0\end{cases}
$$

For $0 \leq \alpha<\beta$, the inclusion $\mathcal{K}_{\infty}^{\alpha}=i m\left(Q_{\infty}^{(\alpha)}\right)^{1 / 2} \supset i m\left(Q_{\infty}^{(\beta)}\right)^{1 / 2}=\mathcal{K}_{\infty}^{\beta}$ always holds, so we have equality if and only if $i m\left(Q_{\infty}^{(\alpha)}\right)^{1 / 2} \subset i m\left(Q_{\infty}^{(\beta)}\right)^{1 / 2}$. Since, clearly, $\left(Q_{\infty}^{(\alpha)}\right)^{1 / 2}$ is the multiplication operator by the function $g_{\alpha}^{1 / 2}$, equality holds if and only if $\sup _{x \in \mathbb{R}} g_{\alpha}(x) / g_{\beta}(x)<\infty$. Taking into account the previous formulae, one concludes that this holds if and only if $1<\alpha<\beta$. Therefore, in this example the spaces $\mathcal{K}_{\infty}^{\alpha}$ are all equal for $\alpha>1$, whereas they are all distinct for $0 \leq \alpha \leq 1$ (and $\mathcal{K}_{\infty}^{1} \supsetneq \mathcal{K}_{\infty}^{\alpha}$ if $\left.\alpha>1\right)$.

The number $\alpha_{0}$ in the statement of Theorem 4.1 is equal to 1 .

Remark 5.6. Let us change the definition of the operator $A$ by subtracting the identity operator; namely, we define the semigroup $\left(e^{t A}\right)$ setting

$$
e^{t A} f(x)=e^{-t} f(x-t), \quad t \geq 0, x \in \mathbb{R} .
$$

Then $\left(e^{t A}\right)$ clearly satisfies $\left\|e^{t A}\right\| \leq e^{-t}$ and so it is exponentially stable. In this case the value of $\alpha_{0}$ is changed to $\alpha_{0}=0$.

5.4. Case (ii) of Theorem 4.1 with $\alpha_{0}=\infty$. We start with some preliminary considerations. Inspection of the statement of Theorem 4.1 shows that if case (i) occurs or if case (ii) occurs with $\alpha_{0}<\infty$, then there exist $\alpha>0$ and $r>0$ such that $\mathcal{K}_{\infty}^{\alpha}=\mathcal{K}_{r}$. Since, as already noted, $\mathcal{K}_{r} \subset \mathcal{K}_{s} \subset \mathcal{K}_{\infty}^{\alpha}$ for $r<s$, it follows that in these cases the spaces $\mathcal{K}_{t}$ coincide for all values of $t$ large enough. Therefore, in order to find a situation where $\alpha_{0}=\infty$, it suffices to construct an example where $\mathcal{K}_{t}$ are all distinct for $t>0$.

The example that follows was communicated to us by Zabczyk.

We take $H=L^{2}([0, \infty))$ and let the operators $e^{t A}$ be the right shift operators

$$
e^{t A} f(x)= \begin{cases}f(x-t), & t \geq 0, x \geq t \\ 0, & t>0, x<t .\end{cases}
$$

Next we denote by $b$ the characteristic function of the interval $[0,1]$ (note that $b \in H$ ); we take $U=\mathbb{R}$ and define the operator $B$ as the rank one operator: $B v=b v, v \in \mathbb{R}$. Since, for every $t>0$,

$$
\mathcal{K}_{t}=i m \mathcal{L}_{t}=\left\{\int_{0}^{t} e^{(t-s) A} b u(s) d s: u \in L^{2}([0, t])\right\},
$$

it can be easily verified that the closure of $\mathcal{K}_{t}$ in $H$, denoted $\overline{\mathcal{K}_{t}}$, is the closure of the linear span of

$$
\left\{e^{r A} b: r \in[0, t]\right\}
$$


It follows that elements of $\overline{\mathcal{K}_{t}}$ have supports contained in the interval $[0,1+t]$ and, moreover, $\overline{\mathcal{K}_{t}}$ contains functions which are nonzero in a left neighborhood of $t$. Therefore, the spaces $\overline{\mathcal{K}}_{t}$ are distinct for different values of $t$, and so the spaces $\mathcal{K}_{t}, t>0$, are also all distinct.

6. Applications to the Ornstein-Uhlenbeck process. As explained in the introduction, one of the motivations for studying the reachability spaces $\mathcal{K}_{\infty}$ introduced above is their probabilistic interpretation. This is the subject of the present section.

Let us consider the following stochastic equation:

$$
\left\{\begin{array}{l}
d X(t)=A X(t) d t+B d W(t), \quad t \geq 0, \\
X(0)=X_{0}
\end{array}\right.
$$

We assume that $H$ and $U$ are real separable Hilbert spaces, $A$ is the generator of a strongly continuous semigroup $\left\{e^{t A}, t \geq 0\right\}$ of bounded linear operators in $H$, and $B$ is a bounded linear operator from $U$ to $H$. We assume that we are also given a probability space $(\Omega, \mathcal{F}, \mathbb{P})$, endowed with a filtration $\left\{\mathcal{F}_{t}, t \geq 0\right\}$ satisfying the usual conditions, and a cylindrical $\left(\mathcal{F}_{t}\right)$-Wiener process $\{W(t) t \geq 0\}$ defined on $\Omega$ with values in $U$; see, e.g., [8]. $X_{0}$ is an $\mathcal{F}_{0}$-measurable random variable with values in $H$. We also assume that the gramian operators

$$
Q_{t} h=\int_{0}^{t} e^{s A} B B^{*} e^{s A^{*}} h d s, \quad h \in H, t>0,
$$

introduced above, have finite trace.

Under these assumptions, the solution of (6.1) is defined as the stochastic process with values in $H$ :

$$
X(t)=e^{t A} X_{0}+\int_{0}^{t} e^{(t-s) A} B d W(s), \quad t \geq 0
$$

The integral occurring in (6.2) is the Itô stochastic integral and, for every $t$, it defines a random variable with values in $H$ because of the finite trace condition Trace $Q_{t}<\infty$. Moreover, if $X_{0}$ is gaussian, then the process $X$ is also gaussian. It is called the (nonsymmetric) Ornstein-Uhlenbeck process. For a detailed exposition of these facts, we refer to [8].

A basic problem is to investigate properties of invariant measures for the process $X$, i.e., Borel probability measures $\nu$ on $H$ such that, if $X_{0}$ has distribution $\nu$, then also $X(t)$ has distribution $\nu$ for every $t$. Invariant measures are known to exist if and only if

$$
\sup _{t>0} \text { Trace } Q_{t}<\infty
$$

In this case, one invariant measure is the gaussian measure $\mu$ on $H$ having zero mean and covariance equal to the extended gramian operator $Q_{\infty}$ introduced above:

$$
Q_{\infty} h=\int_{0}^{\infty} e^{s A} B B^{*} e^{s A^{*}} h d s, \quad h \in H .
$$

Under some additional assumptions, for instance if $A$ is exponentially stable, $\mu$ is the unique invariant measure. For the proof of these facts, we still refer the reader to [8]. 
Remark 6.1. If (6.3) holds, then it is easy to show that condition (3.1) also holds (in particular, Hypothesis 3.1 is satisfied) and the operator $Q_{\infty}$ has finite trace.

Associated to the centered gaussian measure $\mu$ is the so-called Cameron-Martin space (see, e.g., [1]), which coincides with the image of $Q_{\infty}^{1 / 2}$ and hence with the space of reachable states $\mathcal{K}_{\infty}$ introduced in the previous sections.

We address the problem to study the behavior of the Cameron-Martin space under perturbation of the Ornstein-Uhlenbeck process. Thus, for $\alpha \geq 0$, we consider the processes $X_{\alpha}$, solution of

$$
\left\{\begin{array}{l}
d X_{\alpha}(t)=(A-\alpha I) X_{\alpha}(t) d t+B d W(t), \quad t \geq 0 \\
X_{\alpha}(0)=X_{0}
\end{array}\right.
$$

and we consider the centered gaussian measures $\mu_{\alpha}$ with covariance operator $Q_{\infty}^{(\alpha)}$ defined in (4.2). It follows from the previous discussion that $\mu_{\alpha}$ is an invariant measure for $X_{\alpha}$ (and it is unique if $A-\alpha$ is exponentially stable) and that the Cameron-Martin space of $\mu_{\alpha}$ coincides with $\mathcal{K}_{\infty}^{\alpha}$.

The following proposition is the main result of this section. It is a direct consequence of Theorem 4.1.

Proposition 6.2. Assume Hypothesis 3.1 and assume (6.3). Let $\mu_{\alpha}, \alpha \geq 0$, be the centered gaussian invariant measures introduced above, and let $\mathcal{K}_{\infty}^{\alpha}$ be their Cameron-Martin spaces. Then all the conclusions of Theorem 4.1 hold true.

Remark 6.3. The occurrence of the various possibilities described in Theorem 4.1 depends on the inequality $\left\|S_{0}^{(\alpha)}(t)\right\|<1$ for various values of $\alpha \geq 0$ and $t>0$ or the equivalent one $i m\left(Q_{\infty}^{(\alpha)}\right)^{1 / 2}=i m Q_{t}^{1 / 2}$ (compare with Proposition 4.3). These conditions play an important role in connection with various regularity properties of the transition semigroup of the Markov process $X_{\alpha}$.

For instance, assume that Hypothesis 3.1 and (6.3) are satisfied, take $\alpha=0$, and assume that $\operatorname{ker} Q_{\infty}=\{0\}$. (This simplifies some of the statements.) The transition semigroup of the Ornstein-Uhlenbeck process, denoted $\left\{R_{t}, t \geq 0\right\}$, can be considered as a strongly continuous contraction semigroup on each space $L^{p}(H, \mu), p \in[1, \infty)$ (the space of Borel measurable functions $\phi: H \rightarrow \mathbb{R}$ such that $\int_{H}|\phi(x)|^{p} \mu(d x)<\infty$, endowed with its usual norm). It is proved in [3, Theorem 2] that the stronger property

$$
\begin{aligned}
\phi \in L^{p}(H, \mu), p \in(1, \infty) \Longrightarrow & R_{t} \phi \in L^{q}(H, \mu) \text { and } \\
& \left\|R_{t} \phi\right\|_{L^{q}(H, \mu)} \leq\|\phi\|_{L^{p}(H, \mu)}
\end{aligned}
$$

for some $q>p$ holds if and only if $i m Q_{\infty}^{1 / 2}=i m Q_{t}^{1 / 2}$. (The value of $q$ depends on $t$ and $p$.) Property (6.5) is called hypercontractivity (at time $t>0$ ). Thus, by Proposition 4.3 and Corollaries 4.5 and 4.7 , hypercontractivity holds at some $t>0$ if and only if case (i) of Theorem 4.1 occurs.

Similar considerations relate the inequalities $\left\|S_{0}^{(\alpha)}(t)\right\|<1$ (or the equalities $\left.i m\left(Q_{\infty}^{(\alpha)}\right)^{1 / 2}=i m Q_{t}^{1 / 2}\right)$ to various regularity properties of the transition semigroup $R$, such as compactness, differentiability, and smoothing properties in appropriate function spaces. For further details, we refer the reader to $[3,4,5,6,11,13,14]$.

Acknowledgments. We wish to thank Professors Luciano Pandolfi, Roberto Triggiani, and Jerzy Zabczyk for useful discussions. We also thank the anonymous referees for indicating improvements in section 5 . 


\section{REFERENCES}

[1] V. I. Bogachev, Gaussian Measures, Math. Surveys Monogr. 62, AMS, Providence, RI, 1998.

[2] N. Bouleau and F. Hinsch, Dirichlet Forms and Analysis on Wiener Space, de Gruyter Stud. Math. 14, de Gruyter, Berlin, 1991.

[3] A. Chojnowska-Michalik and B. Goldys, Nonsymmetric Ornstein-Uhlenbeck semigroup as second quantized operator, J. Math. Kyoto Univ., 36 (1996), pp. 481-498.

[4] A. Chojnowska-Michalik and B. GoŁdys, Nonsymmetric Ornstein-Uhlenbeck generators, in Infinite Dimensional Stochastic Analysis (Amsterdam, 1999), Verh. Afd. Natuurkd. 1. Reeks. K. Ned. Akad. Wet. 52, R. Neth. Acad. Arts Sci., Amsterdam, 2000, pp. 99-116.

[5] A. Chojnowska-Michalik and B. Goldys, Generalized Ornstein-Uhlenbeck semigroups: Littlewood-Paley-Stein inequalities and the P. A. Meyer equivalence of norms, J. Funct. Anal., 182 (2001), pp. 243-279.

[6] A. Chojnowska-Michalik AND B. GoŁDYs, On regularity properties of nonsymmetric Ornstein-Uhlenbeck semigroup in $L^{p}$ spaces, Stochastics Stochastics Rep., 59 (1996), pp. $183-209$.

[7] R. F. Curtain and H. Zwart, An Introduction to Infinite-Dimensional Linear Systems Theory, Texts Appl. Math. 21, Springer-Verlag, New York, 1995.

[8] G. Da Prato and J. ZabczyK, Stochastic Equations in Infinite Dimensions, Encyclopedia Math. Appl. 44, Cambridge University Press, Cambridge, UK, 1992.

[9] J. Diestel And J. J. UhL, Vector Measures, Mathematical Surveys 15, AMS, Providence, RI, 1977.

[10] N. Dunford And J. T. Schwartz, Linear Operators 1: General Theory, Interscience, New York, 1958.

[11] M. FuHRman, Hypercontractivity properties of nonsymmetric Ornstein-Uhlenbeck semigroups in Hilbert spaces, Stochastic Anal. Appl., 16 (1998), pp. 241-260.

[12] M. FuHRMAn, Logarithmic derivatives of invariant measure for stochastic differential equations in Hilbert spaces, Stochastics Stochastics Rep., 71 (2001), pp. 269-290.

[13] B. GoŁdys, On analyticity of Ornstein-Uhlenbeck semigroups, Atti Accad. Naz. Lincei Cl. Sci. Fis. Mat. Natur. Rend. Lincei (9) Mat. Appl., 10 (1999), pp. 131-140.

[14] B. GoŁDYs, On Bilinear Forms Related to Ornstein-Uhlenbeck Semigroup on Hilbert Space, manuscript.

[15] S. Hansen And G. Weiss, New results on the operator Carleson measure criterion, IMA J. Math Control Inform. 14 (1997), pp. 3-32.

[16] B. Jacob and H. J. Zwart, Disproof of Two Conjectures of George Weiss, Memorandum Faculteit TW 1546, Universiteit Twente, Enschede, The Netherlands, 2000; also available online from http://www.math.utwente.nl/publications/2000/1546.pdf.

[17] Z. M. Ma AND M. Röckner, Introduction to the Theory of (Non-symmetric) Dirichlet Forms, Springer-Verlag, New York, 1992.

[18] D. L. Russell and G. Weiss, A general necessary condition for exact observability, SIAM J. Control Optim., 32 (1994), pp. 1-23.

[19] O. J. Staffans, Well-posed Linear Systems, Cambridge University Press, Cambridge, UK, to appear; also available online from http://www.abo.fi/ staffans/publ.htm.

[20] G. WEISs, A powerful generalization of the Carleson measure theorem, in Open Problems in Mathematical Systems and Control Theory, V. D. Blondel, E. D. Sontag, M. Vidyasagar, and J. C. Willems, eds., Springer-Verlag, London, 1999, pp. 267-272.

[21] J. ZabCZyK, Mathematical Control Theory: An Introduction, Systems Control Found. Appl., Birkhäuser Boston, Boston, 1982. 